Research paper

\title{
A ready-to-use duplex qPCR to detect Leishmania infantum DNA in naturally infected dogs
}

\author{
Rita de Cássia Pontello Rampazzo ${ }^{\mathrm{a}, \mathrm{b}, 1}$, Manuela da Silva Solcà ${ }^{\mathrm{c}, 1}$, Liliane Celestino Sales Santos ${ }^{\mathrm{d}}$, \\ Lais de Novaes Pereira ${ }^{\mathrm{d}}$, José Carlos Oliveira Guedes Jr. ${ }^{\mathrm{d}}$, Patrícia Sampaio Tavares Veras, ${ }^{\mathrm{c}, \text {, }}$ \\ Deborah Bittencourt Mothé Fraga ${ }^{\mathrm{c}, \mathrm{d}, \mathrm{e}}$, Marco Aurélio Krieger ${ }^{\mathrm{a}, \mathrm{b}}$, Alexandre Dias Tavares Costa ${ }^{\mathrm{a}, \mathrm{b}, *}$ \\ a Instituto Carlos Chagas (ICC), FIOCRUZ-PR, Rua Prof. Algacyr Munhoz Mader, 3775 CIC, 81350-010, Curitiba, Paraná, Brazil \\ b Instituto de Biologia Molecular do Paraná (IBMP), Rua Professor Algacyr Munhoz Mader, 3775 CIC, 81350-010, Curitiba, Paraná, Brazil

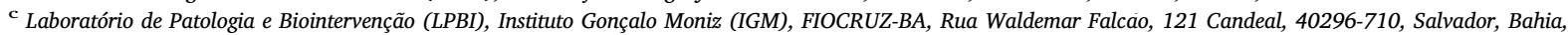 \\ Brazil \\ ${ }^{\mathrm{d}}$ Departamento de Medicina Veterinária Preventiva e Produção Animal, Escola de Medicina Veterinária e Zootecnia, Universidade Federal da Bahia (UFBA), Av. Adhemar \\ de Barros, 500 Ondina, 40170-110, Salvador, Bahia, Brazil \\ e Instituto de Ciência e Tecnologia de Doenças Tropicais, INCT-DT, Salvador, Bahia, Brazil
}

\section{A R T I C L E I N F O}

\section{Keywords:}

Canine visceral leishmaniasis

Diagnosis

Ready-to-use diagnosis technique

Duplex qPCR

Gelified qPCR

\begin{abstract}
A B S T R A C T
Canine visceral leishmaniasis (CVL) is a systemic disease caused by Leishmania infantum. A precise CVL diagnosis would allow for a faster and more specific treatment. Quantitative PCR (qPCR) is a sensitive and specific technique that can diagnose CVL and also monitor parasite load in the animal during the course of the infection or treatment. The aim of this study was to develop a ready-to-use (gelified and freezer-free) duplex qPCR for the identification of infected animals. We combined a new qPCR protocol that detects the canine 18S rRNA gene with an existing protocol for $L$. infantum kDNA detection, creating a duplex qPCR. This duplex method was then developed into a ready-to-use format. The performance of the duplex and singleplex reactions were compared in the traditional format (liquid and freezer-stored). Furthermore, the duplex qPCR performance was compared between the ready-to-use and traditional formats. The singleplex and new duplex qPCR exhibited the same detection limit in the traditional format ( 0.1 parasites/reaction). The ready-to-use format showed a detection limit of 1 parasite/reaction without affecting the reaction efficiency. The performance of the new qPCR protocol in the two formats was assessed using canine tissue samples from 82 dogs in an endemic CVL area that were previously characterized by standard serological and parasitological protocols. Splenic aspirates provided a higher rate of positivity (92.9\%) followed by skin (50\%) and blood (35.7\%). The reported detection limits were observed for all tissues studied. Our results show that the amplification of $L$. infantum kDNA and canine DNA in a single tube, using either the traditional or ready-to-use format, exhibited the same diagnostic performance as amplification of the parasite kDNA alone. The detection of the host gene strengthens the qPCR results by confirming the presence and quality of DNA in the samples and the absence of polymerase inhibitors. The ready-touse duplex qPCR format has many advantages. By joining two qPCR protocols into one, more results can be obtained in the same amount of time with reduced costs and embedded quality control. Reagents are preloaded and stored on the plate, reducing the operator's hands-on time to set up a reaction, as well as decreasing manipulation steps, which reduces the risk of mistakes or contamination. Thus, the ready-to-use duplex format turns qPCR into a robust, easy-to-use tool, which could help increase the availability of qPCR for CVL diagnosis.
\end{abstract}

\section{Introduction}

Leishmania infantum is the agent of visceral leishmaniasis (VL), a neglected tropical disease found throughout Europe and Latin America, and is considered the most severe presentation of the clinical forms of leishmaniasis (Alvar et al., 2012; Desjeux, 2004). Natural transmission occurs via the bite of an infected female phlebotomine (Lainson and Rangel, 2005; Sherlock, 1996).

Dogs are considered the main urban reservoir of L. infantum, mostly due to the high rate of canine infection in endemic areas and intense

\footnotetext{
* Corresponding author at: Instituto Carlos Chagas (ICC), FIOCRUZ-PR, Rua Prof. Algacyr Munhoz Mader, 3775, Curitiba, Paraná, 81350-010, Brazil.

E-mail addresses: alexandre.costa@fiocruz.br, adtcosta@ibmp.org.br (A.D.T. Costa).

1 These authors contributed equally to this work.
} 
parasitism in the skin (Brasil, 2014; Madeira et al., 2009; Margonari et al., 2006; Molina et al., 1994; Oliveira et al., 2001). When infected, dogs exhibit many clinicopathological features that are similar to the human disease (Moreno and Alvar, 2002). Moreover, canine VL (CVL) can present with several degrees of severity and is fatal when not treated (Ciaramella et al., 1997; Koutinas et al., 1999). Due to the importance of the dog as the main urban $L$. infantum reservoir and the fatal course of the disease, a precise CVL diagnosis is essential to implement control measures and prevent death of animals by enabling fast, specific treatment.

Serological tests are widely used in CVL diagnosis (Gomes et al., 2008). However, most dogs exposed to $L$. infantum become infected without demonstrating specific clinical signs and are often lacking serological evidence, making it difficult to diagnose them by conventional serological tests (Baneth et al., 2008; Campino et al., 2000). Additionally, the serological tests used to identify the infection in the canine population may have low sensitivity and specificity, especially in newly infected dogs or in dogs that display a non-specific clinical status (Coura-Vital et al., 2011; Oliva et al., 2006; Otranto et al., 2009). Moreover, some animals present fluctuations in antibody titers during L. infantum infection (Carrera et al., 1996; Rosypal et al., 2005). In endemic areas, it is also common for dogs to have a low parasite load, which results in false-negative results when parasitological tests are used for diagnosis (Gomes et al., 2008). Due to the delayed drop in antibody levels in treated animals, serologic techniques are not adequate for monitoring the efficacy of CVL treatment (Rosypal et al., 2005).

Limitations of the serological and parasitological tests point to the need for developing and implementing more sensitive and specific techniques, such as molecular-based reactions (Grimaldi and Tesh, 1993; Miró et al., 2008; Reale et al., 1999; Solano-Gallego et al., 2001). Molecular techniques allow quantification of the parasite load when quantitative PCR (qPCR) is used (Francino et al., 2006; Manna et al., 2004), either during the course of the natural or experimental infection or after treatment (Maia and Campino, 2008; Manna et al., 2008; Martinez et al., 2011; Pennisi et al., 2005).

Despite the accuracy of qPCR in Leishmania DNA detection, questions regarding the validity of the test results still linger. In particular, there are potential issues relative to the quality of the extracted DNA given that problems in DNA extraction can lead to false-negative results (Goncalves-de-Albuquerque et al., 2014). The main approach to address this issue is to perform a parallel PCR to confirm the integrity of constitutive genes in the host genome. Several housekeeping genes, such as GAPDH, RNase P, and $\beta$-actin, have already been used as internal controls to ensure the DNA template's integrity and prevent false-negative results (Espy et al., 2006; Goncalves-de-Albuquerque et al., 2014; Peleg et al., 2010; Piron et al., 2007). Most of the time, laboratories perform two separate reactions, one for DNA quality control and another to detect the target DNA (Carson et al., 2010; Quaresma et al., 2009; Solcà et al., 2014). Joining these two reactions into one (creating a duplex format) makes the overall reaction cheaper, faster and results in embedded quality control.

Another limitation to the widespread use of a qPCR technique is the requirement for a controlled, stable temperature of $-20{ }^{\circ} \mathrm{C}$ during transport and storage of reagents (i.e., "cold chain"), which steeply increases the costs and in extreme cases precludes use of qPCR as a diagnostic tool (Pai et al., 2012). In recent years, technologies that eliminate the use of $-20{ }^{\circ} \mathrm{C}$ freezers, such as lyophilization and gelification, are currently being evaluated. Among them, gelification is particularly useful due to ease of use in the laboratory as well as introduction into the production line (Iglesias et al., 2014; Rosado et al., 2002; Sun et al., 2013). The gelification process stabilizes qPCR reagents, allowing the complete, ready-to-use reaction to be transported at room temperature and stored at $4{ }^{\circ} \mathrm{C}$ for extended periods (Sun et al., 2013). This format only requires the user to add water and the extracted DNA, preventing mistakes during reagent manipulation and decreasing the hands-on time needed for launching a reaction as well as the possibility of contamination. Therefore, the ready-to-use (gelified and freezer-free) reaction format possesses features that are highly efficient in a routine diagnostic laboratory and can expedite the sample-to-answer process while increasing the robustness of the test.

To the best of our knowledge, this is the first attempt to develop a ready-to-use duplex qPCR for detection of $L$. infantum DNA in dogs. Several conventional PCR protocols are present in the literature targeting DNA from different Leishmania species along with primers to detect sandfly DNA and/or housekeeping genes (de Cassia-Pires et al., 2017; de Pita-Pereira et al., 2008; Rodriguez-Brito et al., 2015). However, none of the protocols describe a ready-to-use or a duplex format using the TaqMan system for detecting $L$. infantum. We developed ready-to-use duplex qPCR to improve the diagnostic protocols used to assess $L$. infantum infection in dogs. The new ready-to-use duplex qPCR was designed to simultaneously identify both a conserved sequence of the host DNA as well as a conserved repetitive sequence of $L$. infantum DNA. We tested the new duplex qPCR protocol for accuracy in the traditional (liquid and freezer-stored) and ready-to-use formats and compared positivity rates in different canine biological tissues to results obtained using serological and parasitological methods.

\section{Materials and methods}

\subsection{Animal and tissue samples}

A convenience sample of 82 dogs was selected from a cross-sectional study conducted in the municipality of Camaçari $\left(12^{\circ} 41^{\prime} 51^{\prime \prime} \mathrm{S} ; 38^{\circ} 19^{\prime}\right.$ 27" W) in Brazil, an endemic area for VL and CVL, during 2015. Three healthy CVL-negative dogs that resided in a non-endemic area for CVL were selected as controls. All procedures were approved by the FIOCRUZ Ethical Committee (Permit Number 007/2013). Canine tissue samples were collected from all 82 dogs as follows: $10 \mathrm{~mL}$ of blood by cephalic puncture, $500 \mu \mathrm{L}$ of splenic aspirate by ultrasound guided aspiration, and a skin fragment from the lateral scapular region using a sterile $3 \mathrm{~mm}$ punch (Kolplast, Brazil). Both skin and splenic samples were collected after performing trichotomy under cutaneous anesthesia with $500 \mu \mathrm{L}$ of $1 \%$ lidocaine hydrochloride (Hypofarma, Brazil). Blood, splenic aspirate, and skin samples were also obtained from CVL-negative dogs and used as negative controls. Blood samples were preserved in EDTA-2Na tubes (Greiner Bio-one, Austria), and all other samples were stored in DNAase-and RNAase-free tubes at $-70{ }^{\circ} \mathrm{C}$ until DNA extraction. DNA extraction of tissue samples was performed using DNeasy Blood \& Tissue Kit (Qiagen, Germany) following the manufacturer's instructions.

\subsection{CVL diagnosis}

For serological CVL diagnosis, we employed the serodiagnostic protocol recommended by the Brazilian Ministry of Health (Brasil, 2014), which consists of the immunochromatographic rapid test DPPLVC (Bio-Manguinhos, Brazil) followed by confirmatory ELISA EIE-LVC (Bio-Manguinhos, Brazil). Serological tests were performed according to the manufacturer's instructions. Parasitological diagnosis was performed by evaluating splenic aspirate cultures as previously described (Barrouin-Melo et al., 2004; Solcà et al., 2012).

\subsection{Standard curve preparation}

L. infantum (MHOM/BR2000/MERIVALDO) DNA was obtained from promastigotes cultivated in Schneider's Complete Medium supplemented with $20 \%$ bovine fetal serum at $24^{\circ} \mathrm{C}$ (Solcà et al., 2014). $L$. infantum promastigotes were counted and sequentially washed with buffer before performing DNA extraction using DNeasy Blood \& Tissue Kit (Qiagen, Hilden, Germany) (Solcà et al., 2014). Extracted DNA was serially diluted (10-fold) in Leishmania-negative tissue-specific DNA 
A

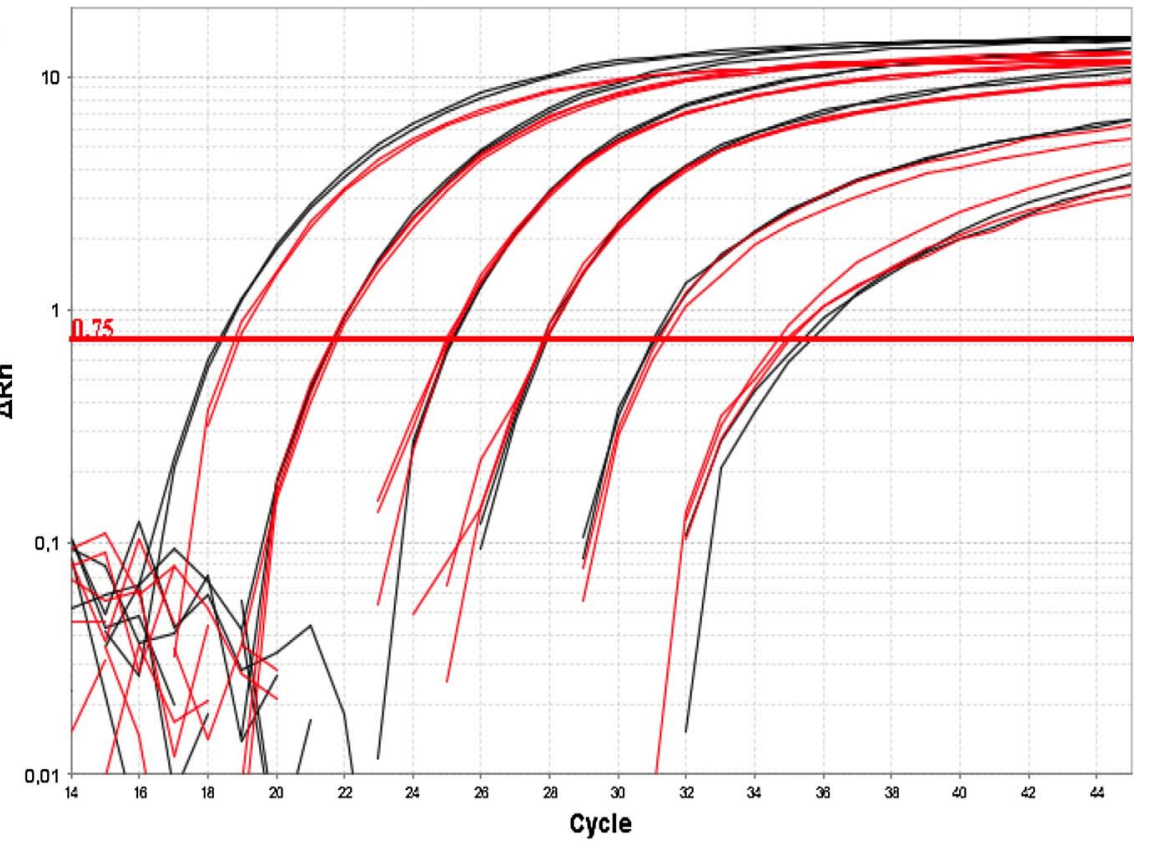

B

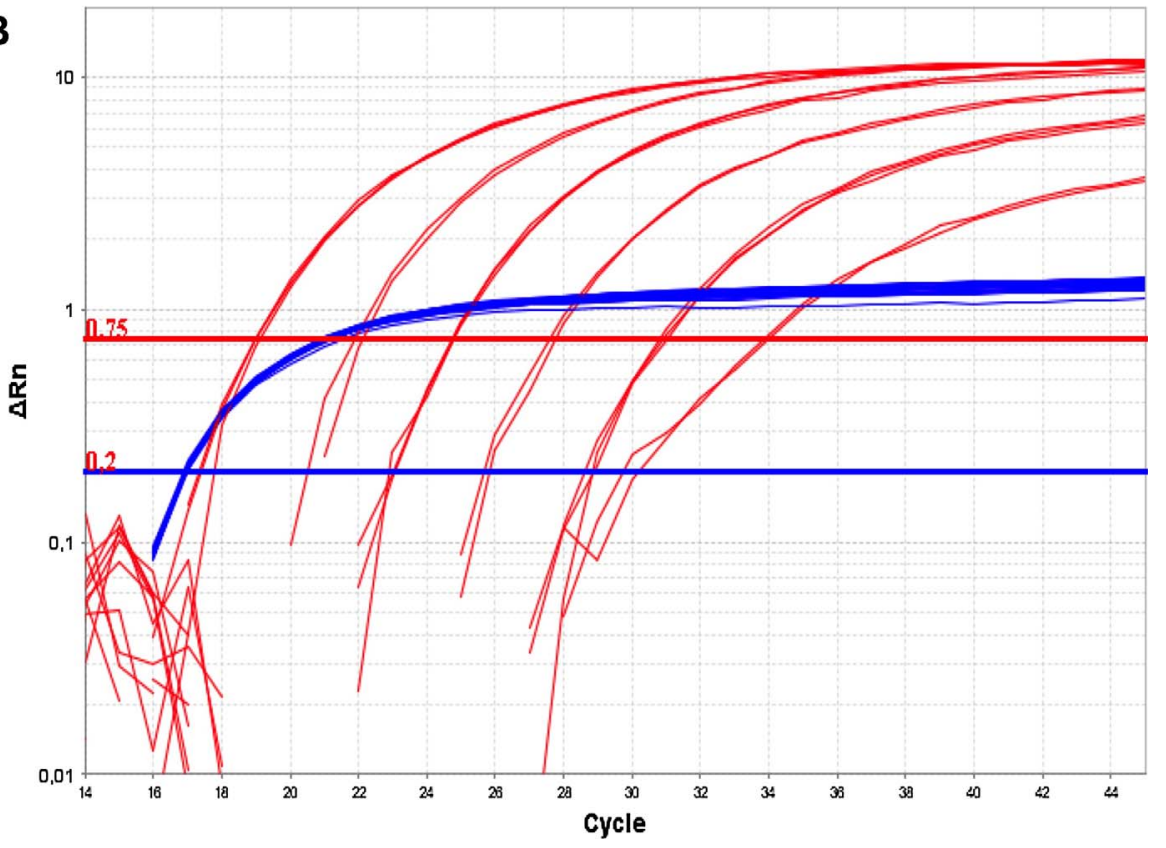

Fig. 1. Reportable ranges for $L$. infantum DNA quantification by qPCR. Panel A shows the amplification plot of 10 -fold serial dilutions of parasite DNA (ranging from $10^{4}$ to 0.1 parasites) detected using traditional singleplex (black lines) or duplex (red lines) qPCR protocols. The thick horizontal red line represents the detection threshold for $L$. infantum DNA detection. Panel B shows only the amplification plot of 10fold serial dilutions of parasite DNA (ranging from $10^{4}$ to 0.1 parasites) in the traditional duplex reaction, depicting the detection of $L$. infantum DNA (red lines) as well as the canine 18S rRNA gene (blue lines). Duplex qPCR was performed using $L$. infantum DNA serially diluted into DNA extracted from Leishmania-negative splenic aspirate. The thick horizontal red line represents the detection threshold for $L$. infantum DNA while the thick horizontal blue line represents the detection threshold for the 18S rRNA DNA. In both singleplex and duplex protocols, each DNA concentration was tested in triplicate. Curves are representative of at least three independent experiments. Mean reaction efficiencies from at least three independent experiments were $98.8 \%$ for the singleplex reaction and $99.8 \%$ for the duplex reaction, all three with a $\mathrm{R}^{2}$ of 0.99 . (For interpretation of the references to colour in this figure legend, the reader is referred to the web version of this article.) obtained from healthy dogs to produce standard curves for each tissue type (blood, splenic aspirate or skin).

\subsection{Duplex $q P C R$ protocol}

We designed a duplex qPCR to simultaneously detect $L$. infantum kinetoplast DNA (kDNA) and a conserved region of the housekeeping gene 18S rRNA ( $\mathrm{gb} \mid \mathrm{DQ} 287955.1$ ). Oligonucleotides for $L$. infantum kDNA detection were: LEISH-1, 5'-AACTTTTCTGGTCCTCCGGGTAG-3'; LEISH-2, 5'-ACCCCCAGTTTCCCGCC-3'; and LEISH-P, 5'-FAMAAAAATGGGTGCAGAAAT-MGB/NFQ-3' (Francino et al., 2006). Oligonucleotides for amplification of the new canine sequence were: 18SCanis_F, 5'-TGCGAATGGCTCATTAAATC-3'; 18SCanis_R, 5'-CGTCGGCATGTATTAGCTCT-3'; and 18SCanis_P, 5'-HEX-TGGTTCCTTTGGTCGCTCGCT-BHQ1-3'.

We tested two methods of storing the qPCR reagents: the traditional format (liquid freezer-stored), where reagents must be thawed prior to use, and a ready-to-use format (gelified, freezer-free), where reagents are stored in the reaction vessel. Traditional duplex qPCR contained $5 \mu \mathrm{L}$ of extracted DNA, Multiplex PCR Mastermix (IBMP/Fiocruz-PR, Brazil), $160 \mathrm{nM}$ each of 18 SCanis_F and 18SCanis_R, $40 \mathrm{nM}$ of 18SCanis_P, $200 \mathrm{nM}$ of LEISH-P, and $900 \mathrm{nM}$ each of LEISH-1 and LEISH-2. Ready-to-use duplex qPCR were produced by mixing qPCR reagents (enzymes, buffer, salts, nucleotides, primers, and probes) with a gelification solution, which was then submitted to vacuum under the same concentrations (Iglesias et al., 2014; Rosado et al., 2002; Sun et al., 2013). Traditional and ready-to-use reactions were run in an ABI7500 Fast Real-Time PCR System (Life Technologies, USA) set with the following protocol: $1 \times 95^{\circ} \mathrm{C} / 10 \mathrm{~min}$; $45 \mathrm{x}$ [95 $\left.{ }^{\circ} \mathrm{C} / 15 \mathrm{~s}, 60^{\circ} \mathrm{C} / 60 \mathrm{~s}\right]$. Reactions were performed in triplicate, and data are expressed as cycle threshold (Ct) mean values. Positivity for $L$. infantum DNA in the qPCR duplex reaction was determined by $\mathrm{Ct}$ cut-off values obtained using a Receiver Operating Characteristic (ROC) curve analysis for each tissue as previously described (Solcà et al., 2014). Tissue-specific Ct cut-off 
values presenting $100 \%$ specificity and the higher sensitivity values were selected.

\subsection{Statistical analysis}

Results of molecular diagnostic techniques were read without any knowledge of the serological or parasitological results to prevent bias. ROC analysis used to establish the Ct cut-off value, was performed using Graph Pad Prism v.5.0 (Graph Pad Prism Inc., USA). Sensitivity and 95\% confidence intervals (CI) were calculated for each tissue using splenic cultures as the standard reference. Comparison of results for each sample among singleplex and duplex formats, as well as traditional and ready-to-use storage formats was evaluated by the Wilcoxon signed rank sum test. Fisher or $\mathrm{X}^{2}$ tests were used to compare the results from each method between all animals in the study. The Kappa coefficient was calculated for each of the diagnostic methods using splenic cultures as the gold standard with a 95\% CI. Additionally, the Kappa coefficient was calculated between duplex qPCR in different tissues, as well as between duplex qPCR in traditional and ready-to-use formats. The coefficient was used to test agreement between the diagnostic methods, and Kappa results were interpreted according to Landis and Koch (1977): 1.00-0.81 almost perfect, 0.80-0.61 substantial, $0.60-0.41$ moderate, $0.40-0.21$ fair and $\leq 0.20$ slight agreement.

\section{Results}

\subsection{Adaptation and standardization of a $q P C R$ singleplex protocol into a duplex $q P C R$ protocol}

We initially adapted a previously described qPCR singleplex protocol (Francino et al., 2006) into a duplex reaction by concurrently detecting the 18S rRNA gene using traditional storage methods for the reagents. Fig. 1 depicts the reportable range for $L$. infantum DNA quantification by qPCR in both singleplex and duplex formats after standardization. Fig. $1 \mathrm{~A}$ shows that the amplification plot of 10 -fold serial dilutions of $L$. infantum DNA in the duplex reaction (red lines) is indistinguishable from detection of $L$. infantum DNA alone in a singleplex reaction (black lines). Fig. 1B shows the amplification of serially diluted $L$. infantum DNA (red lines) concomitant to detection of the constitutive 18S rRNA gene (blue lines). It is noteworthy that detection of the highly abundant 18S rRNA gene did not affect the detection of $L$. infantum DNA, even in very low amounts (i.e., less than one parasitic genome).

\subsection{Evaluation of the detection limit of the traditional duplex $q P C R$ in different canine tissues}

Next, we evaluated the detection limit of the traditional duplex qPCR when used for $L$. infantum DNA detection in different canine tissues. Fig. 2 shows the reportable dynamic range for $L$. infantum DNA quantification by qPCR when parasitic DNA was diluted in DNA extracted from Leishmania-negative blood, skin, or splenic aspirates. Reactions performed in all three DNA matrices exhibited successful linear detection of up to 0.1 parasites/reaction. It should be noted that the duplex reaction was able to stochastically detect 0.05 parasites per reaction (stars).

\subsection{Determination of a Ct cut-off value to evaluate $q P C R$ sample positivity}

We used standardized duplex qPCR to analyze DNA samples from dogs previously diagnosed as positive and negative for CVL and to perform ROC analysis. This analysis determined Ct cut-off values for evaluating QPCR positivity in order to maximize the sensitivity and specificity of the reaction. Cut-off values for blood samples were established at Ct 40.42 , with prediction rates of $91.67 \%$ sensitivity (CI $61.52 \%-100 \%$ ) and $100 \%$ specificity (CI 71.5\%-100\%); for skin

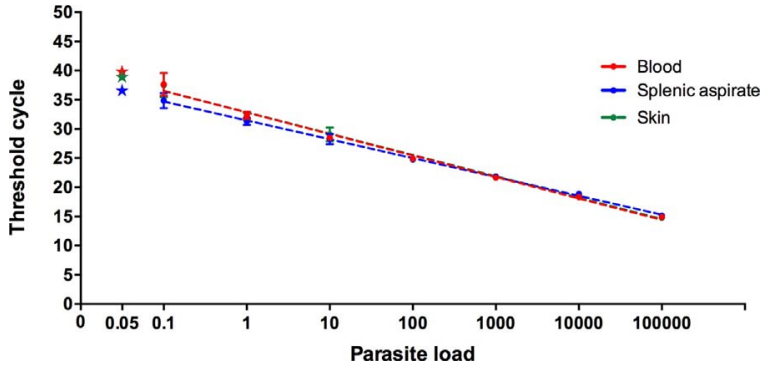

Fig. 2. Comparison of detection limit between $L$. infantum DNA from blood, skin, or splenic aspirates. Ct values obtained for traditional duplex qPCR are plotted against known concentrations of parasite DNA (ranging from $10^{5}$ to 0.1 parasites) diluted in Leishmania-negative DNA from blood, skin, or splenic aspirates. DNA representing 0.05 parasites were stochastically detected and are shown as stars outside the linear regression. Mean reaction efficiencies from at least three independent experiments were $96.8 \%$ for blood and skin samples and $105.9 \%$ for splenic aspirate samples, all three with a $\mathrm{R}^{2}$ of 0.99 .

samples, a Ct cut-off value of 40.0 provided prediction rates of $100 \%$ sensitivity (CI 73.5\%-100\%) and 100\% specificity (CI 69.2\%-100\%); finally, in splenic aspirate samples, the Ct cut-off value of 38.2 corresponded to prediction rates of $91.67 \%$ sensitivity (CI $61.5 \%-99.8 \%$ ) and $100 \%$ specificity (CI 73.5\%-100\%). ROC analysis for the three tissues revealed an area under the curve of 1.0, indicating a high probability $(p<0.001)$ that any randomly chosen positive sample would be correctly classified.

\subsection{Comparison of the performance of the traditional duplex qPCR to other} techniques for CVL diagnosis

We used the convenience sample of 82 dogs to compare the traditional duplex qPCR to other techniques for CVL diagnosis. All dogs were characterized by splenic aspirate cultures, through which 42 were considered positive and 40 were negative. Results from each diagnostic technique are compared in Table 1. qPCR of splenic aspirate samples detected $L$. infantum DNA in $92.9 \%$ of positive animals, while qPCR of skin and blood samples detected $L$. infantum DNA in $50 \%$ and $35.7 \%$ of positive animals, respectively. Statistically significant differences $(p<0.05)$ were observed between the results of splenic aspirate qPCR and all other methods. Among dogs with negative splenic culture tests, qPCR from splenic aspirate samples was able to detect $L$. infantum DNA in $45 \%$ of samples, while qPCR from skin and blood detected DNA in $42.5 \%$ and $27.5 \%$ of samples. No differences were observed between the results of the different diagnostic techniques among animals with negative splenic cultures $(p>0.05)$.

The Kappa coefficient was calculated to measure the agreement index between the gold standard and each of the diagnostic methods tested (Table 1). Kappa coefficients were found to be 0.48 (moderate)

Table 1

Positive results of CVL diagnostic methods performed on samples from 82 dogs, which were divided into two groups according to splenic culture results.

\begin{tabular}{llll}
\hline \multirow{2}{*}{ Diagnostic method } & \multicolumn{2}{l}{ Positivity n (\%) } \\
\cline { 2 - 4 } & \multicolumn{2}{l}{ Splenic culture } \\
\cline { 2 - 4 } & Kappa $^{\#}$ & Positive (n $=42)$ & Negative (n $=40)$ \\
\hline qPCR Splenic aspirate & 0.48 & $39(92.9 \%)$ & $18(45 \%)$ \\
qPCR Skin & 0.07 & $21(50 \%)^{*}$ & $17(42.5 \%)$ \\
qPCR Blood & 0.08 & $15(35.7 \%)^{*}$ & $11(27.5 \%)$ \\
DPP CVL & 0.22 & $30(71.4 \%)^{*}$ & $20(50 \%)$ \\
EIE $^{*}$ CVL & 0.30 & $23(54.8 \%)^{*}$ & $10(25 \%)$ \\
\end{tabular}

\footnotetext{
\# Splenic culture was used as the "gold standard" for Kappa coefficient calculation.

* Statistically different from qPCR splenic aspirate results in the same column by $X^{2}$ $(p<0.05)$.
} 

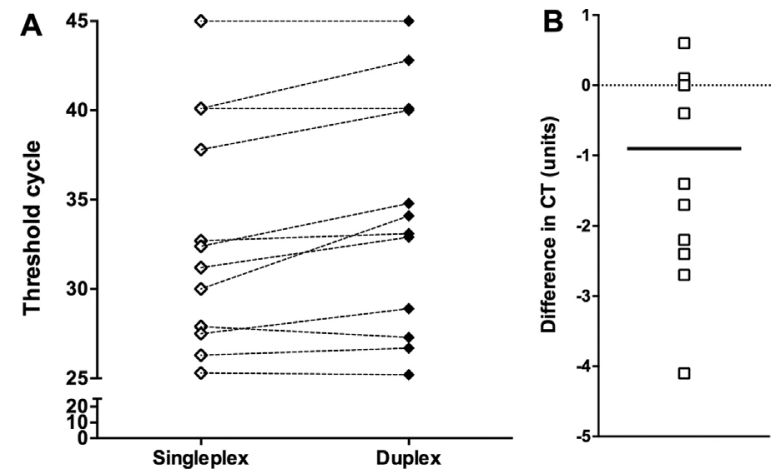

Fig. 3. Comparison of threshold cycles between the traditional singleplex and duplex qPCR for detection of $L$. infantum DNA. Panel A shows the corresponding difference in $\mathrm{Ct}$ values obtained from $\mathrm{QPCR}$ analysis of individual samples tested by the traditional singleplex protocol (empty rhombus) or the traditional duplex protocol (full black rhombus). The same sample was tested independently by both protocols, and the threshold cycles were plotted and connected by dotted lines. Panel B plots the differences between Ct values (empty squares) from the same sample using traditional singleplex or duplex reactions, showing that the mean difference is less than $1 \mathrm{Ct}$ (thick horizontal line). Comparison among singleplex and duplex results for each individual was evaluated by Wilcoxon signed rank sum test, revealing no statistical difference $(p>0.05)$.

for splenic aspirate qPCR and 0.22 and 0.30 (fair) for the serological tests DPP ${ }^{\circ}$ CVL and EIE $^{\circ}$ CVL, respectively. Skin and blood qPCR presented a slight agreement with splenic cultures as indicated by Kappa results of 0.07 and 0.08 , respectively. In addition, we evaluated the agreement between the results of traditional duplex qPCR using different tissues. We found a fair agreement when comparing splenic samples to skin samples (0.22), as well as between skin and blood samples (0.25). Agreement among splenic and blood samples was only slight (0.04).

After analyzing the duplex qPCR results from 82 dogs, Ct intervals for the detection of the conserved canine region for each tissue were established as follows: 15.9-19.9 for blood, 16.6-19.0 for skin, and 17.5-19.8 for splenic aspirate samples, demonstrating that all samples were considered suitable for DNA testing using qPCR.

\subsection{Evaluation of sensitivity variations in the traditional duplex $q P C R$ compared to the qPCR singleplex protocol}

We performed a direct comparison between the Ct of $L$. infantum DNA in randomly selected canine tissue samples analyzed with the singleplex or the duplex reaction using the traditional format. Fig. 3A shows that some samples were detected at a higher $\mathrm{Ct}$ in the duplex format, suggesting a loss of sensitivity to some extent. However, the difference between reactions is negligible, averaging slightly lower mean $\mathrm{Ct}$ values $(<1 \mathrm{Ct})$ with the singleplex relative to the duplex protocol ( $p=0.0248$ ) (Fig. 3B). It is important to highlight that the observed difference did not affect qPCR positivity in this study, irrespective of the protocol used $(p=1.0)$. All samples that were detected as positive by the singleplex reaction were also detected as positive using the duplex reaction.

\subsection{Standardization of the ready-to-use duplex $q P C R$ and comparison to the traditional format performance}

We applied gelification technology to the qPCR reagents and stored them in a ready-to-use format, pre-loaded onto plates inside the production facility. Fig. 4 shows that the reportable range for linear $L$. infantum DNA detection was not significantly affected by the gelification process (Fig. 4A). Although the observed limit of detection for the ready-to-use reaction was 1.0 parasites/reaction, we stochastically detected 0.1 parasites/reaction outside the reaction's linear range. Our results show that gelified $\mathrm{qPCR}$ for detection of $L$. infantum DNA exhibits a similar and relevant linear detection limit as the traditional format, regardless of the tissue type used for sample DNA. Parameters such as amplification efficiency and linearity $\left(R^{2}\right)$ for the gelified duplex qPCR were similar to those obtained for the liquid reaction format (Fig. 4A). Parallel tests of the traditional versus the ready-to-use qPCR format using the same samples showed no meaningful difference (Fig. 4B). Although detection in some samples was affected by the gelification process, on average the delay was less than $1 \mathrm{Ct}$ and reaction performance was not influenced. Fig. 4C summarizes the $\mathrm{Ct}$ differences for the detections in Fig. 4B. Additionally, evaluation of the Kappa coefficient index between the results of traditional and ready-to-use duplex qPCR using different tissues showed perfect agreement (1.00).

\section{Discussion}

A new duplex qPCR protocol for detection of the target (Leishmania DNA) that concomitantly detects a host (canine) control gene is presented herein. The novel development combines a published reaction for detection of $L$. infantum kDNA (Francino et al., 2006) with a new reaction designed for concomitant detection of the canine 18S rRNA gene.

Good performance in a singleplex qPCR protocol is not a guarantee that a corresponding duplex format will maintain the same efficiency, accuracy, and limit of detection (Ferrie et al., 1992; Peleg et al., 2010). Loss of sensitivity and specificity may be due to competition of targets for the enzyme, unspecific interactions among the oligonucleotides or with genomic targets, or the use of identical temperature conditions for both simultaneous reactions. Additionally, avoidance of different contaminating products or primer-probe dimers is also of concern in multiplex reactions (Markoulatos et al., 2002). In sum, the main issue in developing multiplex PCR assays is the difficulty in adjusting two different reactions that occur simultaneously without losing efficiency, accuracy, or sensitivity.

The newly developed traditional qPCR duplex format did not affect the analytical sensitivity of $L$. infantum DNA amplification, presenting the same detection limit of 0.1 parasites/reaction as the singleplex reaction format (Fig. 1). Detection of less than one parasite per reaction is possible because the target gene is present in multiple copies in the genome (Lachaud et al., 2002) and is thus dissolved into solution when the parasites are lysed. For Leishmania spp., the target gene is present at approximately 50 copies per parasite genome (Lachaud et al., 2002).

Successful output of qPCR is directly correlated to the quality of the sample and/or its extracted DNA. One straightforward strategy to simultaneously control the quality of the sample and overall quality of the system and reagents is to amplify a housekeeping gene (Espy et al., 2006; Goncalves-de-Albuquerque et al., 2014; Melo et al., 2015; Peleg et al., 2010; Piron et al., 2007). Herein, we show that the duplex protocol was successfully used for simultaneous detection of $L$. infantum and canine DNA targets in blood, skin, and splenic aspirate samples. It is important to highlight that detection of the highly abundant $18 \mathrm{~S}$ gene did not affect detection of $L$. infantum kDNA, even when the latter was present in much lower amounts.

Blood and skin are known to contain qPCR inhibitors, such as hemoglobin, serum albumin, melanin, and collagen (Giambernardi et al., 1998; Opel et al., 2010; Schrader et al., 2012), which are most likely removed during nucleic acid extraction. In our samples, $18 \mathrm{~S}$ rRNA gene detection was within the expected ranges, demonstrating the quality of the DNA and efficiency of the extraction process in removing qPCR inhibitors (Schrader et al., 2012). We did not observe any meaningful difference between traditional or ready-to-use format for qPCR performed using samples originated from the spleen, blood, or skin. Therefore, when we consider qPCR efficiency and limit of detection, blood, skin, or splenic aspirates are equally good sources for detection of $L$. infantum DNA.

A more in depth analysis of the convenience sample of 82 naturally infected dogs showed that splenic aspirates are the most efficient tissue 

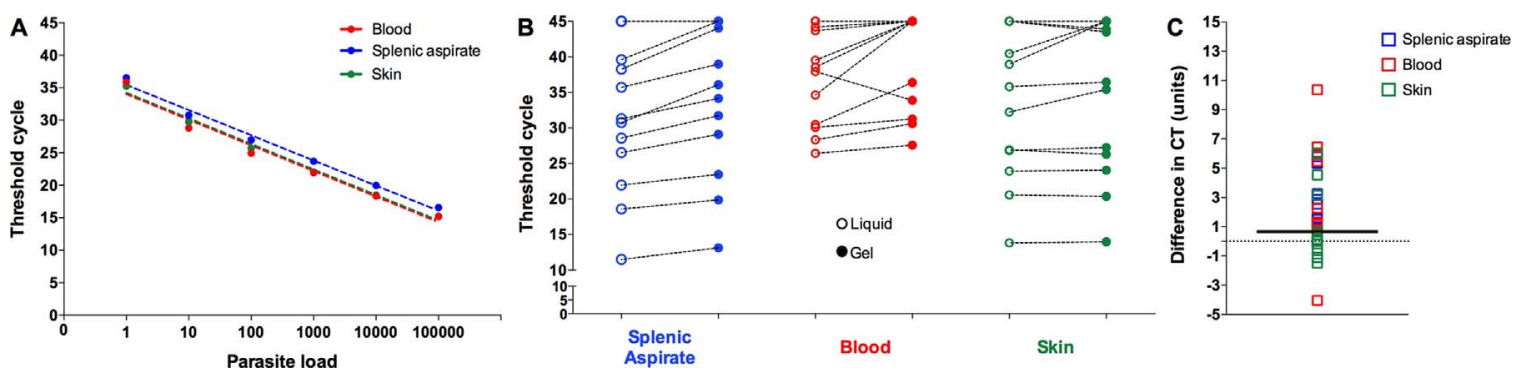

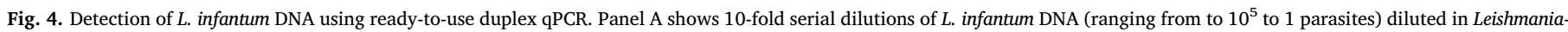

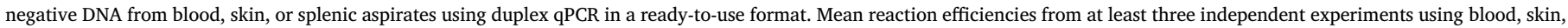

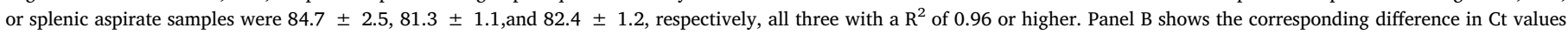

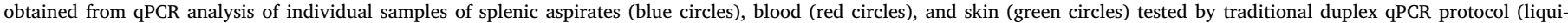

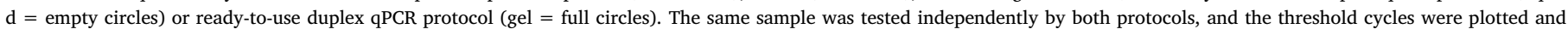

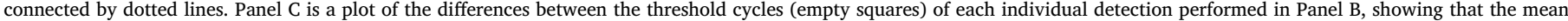

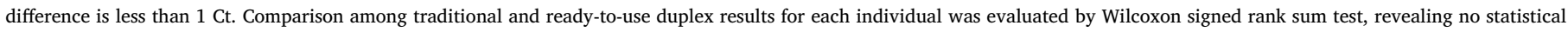
difference $(p>0.05)$. (For interpretation of the references to colour in this figure legend, the reader is referred to the web version of this article.)

for detecting $L$. infantum infection by qPCR. Similar results were also observed in others studies (Solcà et al., 2014). In the present study, we found a moderate agreement between the results of splenic traditional duplex qPCR and splenic cultures, but this is mainly due to the lack of sensitivity of splenic cultures used as the gold standard (Solcà et al., 2014). Splenic aspirates should be preferred over blood or skin if proper collection is possible, such as in clinical practice or research facilities. We believe that the parasite's known splenic tropism is the best explanation for higher positivity in splenic samples (Barrouin-Melo et al., 2006; Reis et al., 2006; Saldarriaga et al., 2006). However, skin should be considered a viable alternative tissue in environments with little-tono infrastructure or unskilled personnel, mainly because the collection procedure is less invasive and requires less technical skill than splenic aspiration. Since skin lesions are known to contain live parasites, this tissue should be considered when skin lesions are present (Aslan et al., 2016).

Although qPCR is a powerful and sensitive technique, its complexity in preparing the reaction is a critical point and a major hurdle for its adoption as a routine diagnostic tool. This difficulty is particularly true for VL and other neglected diseases because they are usually present in countries with insufficient lab infrastructure. Moreover, qPCR reagents require maintenance of freezing temperatures during transportation/ storage, which is not an easy task in tropical countries. Therefore, almost as a sine qua non condition, a good diagnostic test for neglected tropical diseases should not be influenced by transportation/storage temperature (Pai et al., 2012). Interestingly, the gelified format has previously been used to store $\mathrm{qPCR}$ reagents at $4{ }^{\circ} \mathrm{C}$ for up to 12 months or at $22^{\circ} \mathrm{C}$ for $1-3$ months without significant loss of sensitivity (Iglesias et al., 2014; Rosado et al., 2002; Sun et al., 2013). Additionally, the ready-to-use format is advantageous because it decreases the manipulation steps to set up a reaction and minimizes the risk of operator mistakes by pre-loading all reagents directly into the reaction plate. Plasmodium spp. and Campylobacter spp. are examples of pathological organisms whose detection has been facilitated by pre-storing the gelified qPCR reagents directly on the reaction vessel (Iglesias et al., 2014; Sun et al., 2013). Our results show that gelified qPCR for detection of $L$. infantum DNA exhibits a similar relevant linear detection limit as the traditional format, irrespective of the tissue type used for sample DNA. This is a significant step towards increasing the use of qPCR for CVL diagnosis. We are currently performing long term storage studies on gelified $L$. infantum detection.

We suggest that QPCR could be used as a confirmatory test for CVL diagnosis in veterinary clinics, particularly in this ready-to-use duplex format. Previous studies (Manna et al., 2006; Solcà et al., 2014) have shown how the parasite load is an important biomarker for infection monitoring. In this sense, qPCR is a very appropriate tool for clinical assessment of dogs treated for $L$. infantum infection as well as in CVL research. Precise quantification of the parasite load in experimental infections may allow for advances in studies about the pathogenesis of the disease, as well as reliable comparisons in studies evaluating the efficacy of new anti-Leishmania drugs (Roura et al., 2013). Additionally, duplex qPCR has applicability for the diagnostic evaluation of VL in humans, especially in infected individuals who are also HIV positive and do not produce specific antibodies against the parasite, which makes diagnosis difficult using serological tests (Alvar et al., 1997).

In summary, we developed a duplex qPCR for detection of $L$. infantum DNA in canine blood, skin, or splenic aspirate samples that is as sensitive as its singleplex counterpart. Also, we present a ready-to-use gelified freezer-free duplex qPCR format that detects clinically relevant concentrations of parasites and only requires pre-loading the DNA samples directly into the reaction vessel, thereby decreasing manipulation of reagents and minimizing the risk of operator mistakes. In veterinary practice and research studies, more specific techniques such as QPCR could and should be applied as follow-up tests during drug treatment. The protocol presented herein would greatly enhance the accuracy of CVL diagnosis and lead to substantial improvements in control efforts.

\section{Conflict of interest statement}

The authors declare no potential conflicts of interest with respect to the research, authorship, publication of this article, and/or financial and personal relationships that could inappropriately influence this work.

\section{Acknowledgments}

The authors would like to thank the Secretary of Health of Camaçari and the staff of the Zoonosis Control Center of Camaçari for their assistance in conducting the study. We would especially like to thank Gilmar Cerqueira Pereira and health agents for their help in the endemic area. This work was partially funded by FINEP (\#01.13.0283.00 to M.A.K). FINEP played no role in designing the study, data collection, analysis, and interpretation, or in writing or submission of the manuscript. This manuscript has been edited by native English-speaking experts from BioMed Proofreading ${ }^{\circledast}$ LLC.

\section{References}

Alvar, J., Canavate, C., Gutierrez-Solar, B., Jimenez, M., Laguna, F., Lopez-Velez, R., Molina, R., Moreno, J., 1997. Leishmania and human immunodeficiency virus coinfection: the first 10 years. Clin. Microbiol. Rev. 10, 298-319.

Alvar, J., Velez, I.D., Bern, C., Herrero, M., Desjeux, P., Cano, J., Jannin, J., den Boer, M., W.H.O.L.C. Team, 2012. Leishmaniasis worldwide and global estimates of its incidence. PLoS One 7, e35671. 
Aslan, H., Oliveira, F., Meneses, C., Castrovinci, P., Gomes, R., Teixeira, C., Derenge, C.A., Orandle, M., Gradoni, L., Oliva, G., Fischer, L., Valenzuela, J.G., Kamhawi, S., 2016. New insights into the transmissibility of Leishmania infantum from dogs to sand flies: experimental vector-transmission reveals persistent parasite depots at bite sites. J. Infect. Dis. 213, 1752-1761.

Baneth, G., Koutinas, A.F., Solano-Gallego, L., Bourdeau, P., Ferrer, L., 2008. Canine leishmaniosis-new concepts and insights on an expanding zoonosis: part one. Trends Parasitol. 24, 324-330.

Barrouin-Melo, S.M., Larangeira, D.F., Trigo, J., Aguiar, P.H., dos-Santos, W.L., Pontes-deCarvalho, L., 2004. Comparison between splenic and lymph node aspirations as sampling methods for the parasitological detection of Leishmania chagasi infection in dogs. Mem. Inst. Oswaldo Cruz 99, 195-197.

Barrouin-Melo, S.M., Larangeira, D.F., de Andrade Filho, F.A., Trigo, J., Juliao, F.S., Franke, C.R., Palis Aguiar, P.H., Conrado dos-Santos, W.L., Pontes-de-Carvalho, L., 2006. Can spleen aspirations be safely used for the parasitological diagnosis of canine visceral leishmaniosis? A study on assymptomatic and polysymptomatic animals. Vet. J. 171, 331-339.

Brasil, 2014. Manual de Vigilância e Controle da Leishmaniose Visceral. (Brasília, Ministério da Saúde). p. 122.

Campino, L., Santos-Gomes, G., Rica Capela, M.J., Cortes, S., Abranches, P., 2000. Infectivity of promastigotes and amastigotes of Leishmania infantum in a canine model for leishmaniosis. Vet. Parasitol. 92, 269-275.

Carrera, L., Fermin, M.L., Tesouro, M., Garcia, P., Rollan, E., Gonzalez, J.L., Mendez, S., Cuquerella, M., Alunda, J.M., 1996. Antibody response in dogs experimentally infected with Leishmania infantum: infection course antigen markers. Exp. Parasitol. 82, 139-146.

Carson, C., Quinnell, R.J., Holden, J., Garcez, L.M., Deborggraeve, S., Courtenay, O., 2010. Comparison of Leishmania OligoC-TesT PCR with conventional and real-time PCR for Diagnosis of canine Leishmania infection. J. Clin. Microbiol. 48, 3325-3330.

Ciaramella, P., Oliva, G., Luna, R.D., Gradoni, L., Ambrosio, R., Cortese, L., Scalone, A., Persechino, A., 1997. A retrospective clinical study of canine leishmaniasis in 150 dogs naturally infected by Leishmania infantum. Vet. Rec. 141, 539-543.

Coura-Vital, W., Marques, M.J., Giunchetti, R.C., Teixeira-Carvalho, A., Moreira, N.D., Vitoriano-Souza, J., Vieira, P.M., Carneiro, C.M., Correa-Oliveira, R., Martins-Filho, O.A., Carneiro, M., Reis, A.B., 2011. Humoral and cellular immune responses in dogs with inapparent natural Leishmania infantum infection. Vet. J. 190, e43-47.

de Cassia-Pires, R., de Melo, M.F., Barbosa, R.D., Roque, A.L., 2017. Multiplex PCR as a tool for the diagnosis of Leishmania spp. kDNA and the gapdh housekeeping gene of mammal hosts. PLoS One 12, e0173922.

de Pita-Pereira, D., Cardoso, M.A., Alves, C.R., Brazil, R.P., Britto, C., 2008. Detection of natural infection in Lutzomyia cruzi and Lutzomyia forattinii (Diptera: psychodidae: Phlebotominae) by Leishmania infantum chagasi in an endemic area of visceral leishmaniasis in Brazil using a PCR multiplex assay. Acta Trop. 107, 66-69.

Desjeux, P., 2004. Leishmaniasis. Nat. Rev. Microbiol. 2, 692.

Espy, M.J., Uhl, J.R., Sloan, L.M., Buckwalter, S.P., Jones, M.F., Vetter, E.A., Yao, J.D., Wengenack, N.L., Rosenblatt, J.E., Cockerill 3rd, F.R., Smith, T.F., 2006. Real-time PCR in clinical microbiology: applications for routine laboratory testing. Clin. Microbiol. Rev. 19, 165-256.

Ferrie, R.M., Schwarz, M.J., Robertson, N.H., Vaudin, S., Super, M., Malone, G., Little, S., 1992. Development, multiplexing, and application of ARMS tests for common mutations in the CFTR gene. Am. J. Hum. Genet. 51, 251-262.

Francino, O., Altet, L., Sanchez-Robert, E., Rodriguez, A., Solano-Gallego, L., Alberola, J., Ferrer, L., Sanchez, A., Roura, X., 2006. Advantages of real-time PCR assay for diagnosis and monitoring of canine leishmaniosis. Vet. Parasitol. 137, 214-221.

Giambernardi, T.A., Rodeck, U., Klebe, R.J., 1998. Bovine serum albumin reverses inhibition of RT-PCR by melanin. Biotechniques 25, 564-566.

Gomes, Y.M., Paiva Cavalcanti, M., Lira, R.A., Abath, F.G., Alves, L.C., 2008. Diagnosis of canine visceral leishmaniasis: biotechnological advances. Vet. J. 175, 45-52.

Goncalves-de-Albuquerque, S.C., Pessoa e Silva, R., de Morais, R.C., Trajano-Silva, L.A., Regis-da-Silva, C.G., Brandao-Filho, S.P., de Paiva-Cavalcanti, M., 2014. Tracking false-negative results in molecular diagnosis: proposal of a triplex-PCR based method for leishmaniasis diagnosis. J. Venom. Anim. Toxins Incl. Trop. Dis. 20, 16.

Grimaldi Jr., G., Tesh, R.B., 1993. Leishmaniases of the New World: current concepts and implications for future research. Clin. Microbiol. Rev. 6, 230-250.

Iglesias, N., Subirats, M., Trevisi, P., Ramirez-Olivencia, G., Castan, P., Puente, S., Toro, C., 2014. Performance of a new gelled nested PCR test for the diagnosis of imported malaria: comparison with microscopy, rapid diagnostic test, and real-time PCR. Parasitol. Res. 113, 2587-2591.

Koutinas, A.F., Polizopoulou, Z.S., Saridomichelakis, M.N., Argyriadis, D., Fytianou, A. Plevraki, K.G., 1999. Clinical considerations on canine visceral leishmaniasis in Greece: a retrospective study of 158 cases (1989-1996). J. Am. Anim. Hosp. Assoc. 35, 376-383.

Lachaud, L., Marchergui-Hammami, S., Chabbert, E., Dereure, J., Dedet, J.P., Bastien, P., 2002. Comparison of six PCR methods using peripheral blood for detection of canine visceral leishmaniasis. J. Clin. Microbiol. 40, 210-215.

Lainson, R., Rangel, E.F., 2005. Lutzomyia longipalpis and the eco-epidemiology of American visceral leishmaniasis, with particular reference to Brazil: a review. Mem. Inst. Oswaldo Cruz 100, 811-827.

Landis, J.R., Koch, G.G., 1977. The measurement of observer agreement for categorical data. Biometrics 159-174.

Madeira, M.F., Figueiredo, F.B., Pinto, A.G., Nascimento, L.D., Furtado, M., MoutaConfort, E., de Paula, C.C., Bogio, A., Gomes, M.C., Bessa, A.M., Passos, S.R., 2009. Parasitological diagnosis of canine visceral leishmaniasis: is intact skin a good target? Res. Vet. Sci. 87, 260-262.

Maia, C., Campino, L., 2008. Methods for diagnosis of canine leishmaniasis and immune response to infection. Vet. Parasitol. 158, 274-287.
Manna, L., Vitale, F., Reale, S., Caracappa, S., Pavone, L.M., Morte, R.D., Cringoli, G., Staiano, N., Gravino, A.E., 2004. Comparison of different tissue sampling for PCRbased diagnosis and follow-up of canine visceral leishmaniosis. Vet. Parasitol. 125, 251-262.

Manna, L., Reale, S., Viola, E., Vitale, F., Manzillo, V.F., Michele, P.L., Caracappa, S., Gravino, A.E., 2006. Leishmania DNA load and cytokine expression levels in asymptomatic naturally infected dogs. Vet. Parasitol. 142, 271-280.

Manna, L., Reale, S., Vitale, F., Picillo, E., Pavone, L.M., Gravino, A.E., 2008. Real-time PCR assay in Leishmania-infected dogs treated with meglumine antimoniate and allopurinol. Vet. J. 177, 279-282.

Margonari, C., Freitas, C.R., Ribeiro, R.C., Moura, A.C., Timbo, M., Gripp, A.H., Pessanha, J.E., Dias, E.S., 2006. Epidemiology of visceral leishmaniasis through spatial analysis, in Belo Horizonte municipality, state of Minas Gerais, Brazil. Mem. Inst. Oswaldo Cruz 101, 31-38.

Markoulatos, P., Siafakas, N., Moncany, M., 2002. Multiplex polymerase chain reaction: a practical approach. J. Clin. Lab. Anal. 16, 47-51.

Martinez, V., Quilez, J., Sanchez, A., Roura, X., Francino, O., Altet, L., 2011. Canine leishmaniasis: the key points for QPCR result interpretation. Parasit. Vectors 4, 57.

Melo, M.F., Moreira, O.C., Tenorio, P., Lorena, V., Lorena-Rezende, I., Junior, W.O., Gomes, Y., Britto, C., 2015. Usefulness of real time PCR to quantify parasite load in serum samples from chronic Chagas disease patients. Parasit. Vectors 8, 154.

Miró, G., Cardoso, L., Pennisi, M.G., Oliva, G., Baneth, G., 2008. Canine leishmaniosis-new concepts and insights on an expanding zoonosis: part two. Trends Parasitol. 24, 371-377.

Molina, R., Amela, C., Nieto, J., San-Andres, M., Gonzalez, F., Castillo, J.A., Lucientes, J., Alvar, J., 1994. Infectivity of dogs naturally infected with Leishmania infantum to colonized Phlebotomus perniciosus. Trans. R. Soc. Trop. Med. Hyg. 88, 491-493.

Moreno, J., Alvar, J., 2002. Canine leishmaniasis: epidemiological risk and the experi mental model. Trends Parasitol. 18, 399-405.

Oliva, G., Scalone, A., Foglia Manzillo, V., Gramiccia, M., Pagano, A., Di Muccio, T., Gradoni, L., 2006. Incidence and time course of Leishmania infantum infections examined by parasitological, serologic, and nested-PCR techniques in a cohort of naive dogs exposed to three consecutive transmission seasons. J. Clin. Microbiol. 44, 1318-1322.

Oliveira, C.D.L., Assunção, R.M., Reis, I.A., Proietti, F.A., 2001. Spatial distribuition of human and canine visceral leishmaniasis in Belo Horizonte Minas Gerais state, Brazil. Cad de Saúde Pública 17, 1231-1239.

Opel, K.L., Chung, D., McCord, B.R., 2010. A study of PCR inhibition mechanisms using real time PCR. J. Forensic Sci. 55, 25-33.

Otranto, D., Paradies, P., de Caprariis, D., Stanneck, D., Testini, G., Grimm, F., Deplazes, P., Capelli, G., 2009. Toward diagnosing Leishmania infantum infection in asymptomatic dogs in an area where leishmaniasis is endemic. Clin. Vaccine Immunol. 16, 337-343.

Pai, N.P., Vadnais, C., Denkinger, C., Engel, N., Pai, M., 2012. Point-of-care testing for infectious diseases: diversity, complexity, and barriers in low- and middle-income countries. PLoS Med. 9, e1001306.

Peleg, O., Baneth, G., Eyal, O., Inbar, J., Harrus, S., 2010. Multiplex real-time qPCR for the detection of Ehrlichia canis and Babesia canis vogeli. Vet. Parasitol. 173, 292-299.

Pennisi, M.G., Reale, S., Giudice, S.L., Masucci, M., Caracappa, S., Vitale, M., Vitale, F. 2005. Real-time PCR in dogs treated for leishmaniasis with allopurinol. Vet. Res, Commun. 29 (Suppl. 2), 301-303.

Piron, M., Fisa, R., Casamitjana, N., Lopez-Chejade, P., Puig, L., Verges, M., Gascon, J., Gomez i Prat, J., Portus, M., Sauleda, S., 2007. Development of a real-time PCR assay for Trypanosoma cruzi detection in blood samples. Acta Trop. 103, 195-200.

Quaresma, P.F., Murta, S.M., Ferreira Ede, C., da Rocha-Lima, A.C., Xavier, A.A., Gontijo, C.M., 2009. Molecular diagnosis of canine visceral leishmaniasis: identification of Leishmania species by PCR-RFLP and quantification of parasite DNA by real-time PCR. Acta Trop. 111, 289-294.

Reale, S., Maxia, L., Vitale, F., Glorioso, N.S., Caracappa, S., Vesco, G., 1999. Detection of Leishmania infantum in dogs by PCR with lymph node aspirates and blood. J. Clin. Microbiol. 37, 2931-2935.

Reis, A.B., Martins-Filho, O.A., Teixeira-Carvalho, A., Carvalho, M.G., Mayrink, W., Franca-Silva, J.C., Giunchetti, R.C., Genaro, O., Correa-Oliveira, R., 2006. Parasite density and impaired biochemical/hematological status are associated with severe clinical aspects of canine visceral leishmaniasis. Res. Vet. Sci. 81, 68-75.

Rodriguez-Brito, S., Camacho, E., Mendoza, M., Nino-Vega, G.A., 2015. Differential identification of Sporothrix spp. and Leishmania spp. by conventional PCR and qPCR in multiplex format. Med. Mycol. 53, 22-27.

Rosado, S., López, P.F., Limones, G., Seiz, A.M., Alberdi, M.D.M., 2002. Method for preparing stabilised reaction mixtures, which are totally or partially dried, comprising at least one enzyme, reaction mixtures and kits containing said mixtures. Organization, W.I.P., (ed.) (Spain).

Rosypal, A.C., Troy, G.C., Duncan, R.B., Zajac, A.M., Lindsay, D.S., 2005. Utility of diagnostic tests used in diagnosis of infection in dogs experimentally inoculated with North American isolate of Leishmania infantum infantum. J. Vet. Intern. Med. 19, 802-809.

Roura, X., Fondati, A., Lubas, G., Gradoni, L., Maroli, M., Oliva, G., Paltrinieri, S., Zatelli, A., Zini, E., 2013. Prognosis and monitoring of leishmaniasis in dogs: a working group report. Vet. J. 198, 43-47.

Saldarriaga, O.A., Travi, B.L., Park, W., Perez, L.E., Melby, P.C., 2006. Immunogenicity of a multicomponent DNA vaccine against visceral leishmaniasis in dogs. Vaccine 24 , 1928-1940.

Schrader, C., Schielke, A., Ellerbroek, L., Johne, R., 2012. PCR inhibitors-occurrence, properties and removal. J. Appl. Microbiol. 113, 1014-1026.

Sherlock, I.A., 1996. Ecological interactions of visceral leishmaniasis in the state of Bahia, Brazil. Mem. Inst. Oswaldo Cruz 91, 671-683. 
Solano-Gallego, L., Morell, P., Arboix, M., Alberola, J., Ferrer, L., 2001. Prevalence of Leishmania infantum infection in dogs living in an area of canine leishmaniasis endemicity using PCR on several tissues and serology. J. Clin. Microbiol. 39, 560-563.

Solcà, M.d.S., Guedes, C.E., Nascimento, E.G., Oliveira, G.G., dos Santos, W.L., Fraga, D.B., Veras, P.S., 2012. Qualitative and quantitative polymerase chain reaction (PCR) for detection of Leishmania in spleen samples from naturally infected dogs. Vet. Parasitol. 184, 133-140.

Solcà, M.S., Bastos, L.A., Guedes, C.E., Bordoni, M., Borja, L.S., Larangeira, D.F., da Silva
Estrela Tuy, P.G., Amorim, L.D., Nascimento, E.G., de Sa Oliveira, G.G., dos-Santos, Fraga, W.L., Veras, D.B.P.S., 2014. Evaluating the accuracy of molecular diagnostic testing for canine visceral leishmaniasis using latent class analysis. PLoS One 9, e103635.

Sun, Y., Hogberg, J., Christine, T., Florian, L., Monsalve, L.G., Rodriguez, S., Cao, C., Wolff, A., Ruano-Lopez, J.M., Bang, D.D., 2013. Pre-storage of gelified reagents in lab-on-a-foil system for rapid nucleic acid analysis. Lab Chip 13, 1509-1514. 